\title{
La distribución de ingresos y rentas en América Latina durante el siglo XX: un estudio inicial
}

\author{
Valpy FitzGerald \\ St Antony's College \\ Oxford
}

\begin{abstract}
Resumen
América Latina es la región del mundo con la mayor desigualdad en la distribución de ingresos. Sin embargo aún no se conocen suficientemente las causas históricas de esta situación. En este artículo se informa sobre la primera tentativa de calcular la distribución de los ingresos en las cinco economías latinoamericanas más importantes a lo largo del siglo XX. La metodología aporta estimaciones de la dispersión de la renta de los factores entre 1900 y 2000 para cuatro niveles de cualificación. Dichas estimaciones pueden emplearse para crear coeficientes de Gini. Se hallaron importantes fluctuaciones de la dispersión a lo largo del tiempo, lo que contradice la estabilidad desde la época colonial que se propugna en algunas publicaciones recientes de instituciones económicas y respalda las conclusiones de historiadores económicos y economistas del desarrollo. Se estudiaron una serie de variables que podrían explicar dichas fluctuaciones: el impacto del comercio internacional, la calidad de la mano de obra y los desequilibrios macroeconómicos. El modelo de estimación resultante explica bien los datos, ya que los tres conjuntos de factores resultaron ser importantes, aunque los efectos medidos fueron distintos en los cinco países. En el artículo se llega a la conclusión de que la composición de la mano de obra en cuanto a niveles de cualificación, además de sustentar las tendencias a largo plazo en la distribución funcional de la renta -como pensaba Kuznets - condiciona las fluctuaciones producidas en reacción a impactos de origen externo.
\end{abstract}

Palabras clave: Historia económica, bienestar y pobreza, demografía, renta y riqueza.

Classificación JEL: I31, N36.

\begin{abstract}
Latin America has the most unequal income distribution of any region in the world, yet its historical causes are poorly understood. This paper reports the first exploratory attempt to compute income distributions for the five leading Latin American economies for the whole twentieth century. The methodology produces estimates of factor income dispersion for four skill groups over 19002000, which can be used to generate Gini coefficients. Large fluctuations in dispersion over time are found: countering claims of stability since the colonial past in the recent economic institutions literature; but supporting the findings of economic historians and development economists. A set of explanatory variables (reflecting the impact of international trade, labour quality and macroeconomic imbalances) are tested. The resulting estimation model explains the data well, with all three sets of drivers proving significant; although the measured effects are different across the five countries. The paper concludes that the skill composition of the workforce not only underpins long run trends in functional income distribution as conceived by Kuznets; but also conditions fluctuations in response to exogenous shocks.
\end{abstract}

Keywords: Economic history, welfare and poverty, demography, income and wealth.

JEL Classification: I31, N36. 


\section{Introducción ${ }^{1}$}

Suele coincidirse en que América Latina tiene la peor distribución de renta de todas las regiones del mundo en este principio del siglo XXI, lo que tiene graves consecuencias en el nivel de vida medio, la incidencia de la pobreza y la exclusión social. ${ }^{2}$ Está claro que las raíces de la desigualdad actual se encuentran en procesos anteriores de desarrollo económico, pero no existen estimaciones de las tendencias a largo plazo de la distribución de la renta que permitan conocer estas raíces.

Una publicación reciente de corte neoinstitucionalista afirma que las causas primigenias de la desigualdad existente en la región se encuentran en el pasado colonial y, más aún, que dicha desigualdad se ha mantenido prácticamente sin cambios. ${ }^{3}$ Este determinismo histórico se expresa en un reciente estudio del Banco Mundial:

La extrema desigualdad surgió poco después de que los europeos comenzaran a colonizar América hace quinientos años y se ha visto reflejada en las instituciones que crearon. Tanto esta desigualdad inicial como las instituciones se vieron influidas en gran medida por las dotaciones de factores que los europeos encontraron en América Central y en Sudamérica, más que en la naturaleza de los propios poderes coloniales. ... La desigualdad extrema se mantuvo en los siglos XIX y XX porque la evolución de las instituciones políticas y económicas tendía a reproducir y reforzar una distribución muy desigual de la riqueza, el capital humano y la influencia política. ${ }^{4}$

Este parecer subyace también en influentes estimaciones de desigualdad mundial a largo plazo como la de Bourguignon y Morrison (2002), quienes se limitan a suponer que la distribución de la renta en América Latina se mantuvo constante entre 1820 y 1950 y apenas ha cambiado a partir de entonces.

Sin embargo, las investigaciones empíricas en profundidad no respaldan esta opinión. Un análisis integral de datos de encuestas domésticas realizado entre 1970 y 1995 por el Banco Interamericano de Desarrollo concluyó que:

las grandes fluctuaciones en la desigualdad agregada de los países latinoamericanos experimentadas en los últimos 26 años son el resultado de grandes redistribuciones de la renta producidas en los países [...] Esta conclusión no concuerda con la idea propuesta por Li et al. (1998) o Deininger y Squire (1996) como un «nuevo hecho estilizado» del desarrollo, según la cual la desigualdad dentro de los distintos países carece relativamente de importancia en comparación con las diferencias existentes entre los países. ${ }^{5}$

\footnotetext{
${ }^{1}$ Es justo agradecer profusamente a Ame Berges por recopilar la serie estadística y a Pablo Astorga por su ayuda con la econometría.

2 Milanovic (2005) obtuvo un coeficiente de Gini para las rentas familiares correspondiente a América Latina en el año 2000 de 0,51, frente al 0,31 de los países de la OCDE, el 0,36 de Asia y el 0,47 de África.

${ }^{3}$ Véanse por ejemplo: Acemoglu, Johnson y Robinson (2002); Engerman, Haber y Sokoloff (2000); Engerman y Sokoloff (1997); Sokoloff y Engerman (2000).

${ }^{4}$ De Ferranti et al. (2004), página 4-1.

${ }^{5}$ Londoño y Szekely (1997), p. 8.
} 
Por otra parte, las estimaciones realizadas por algunos historiadores económicos de la relación entre salarios reales y PIB real per cápita antes de la Segunda Guerra Mundial sugieren que la distribución de la renta en América Latina sufrió grandes fluctuaciones debido a los cambios en el precio de factores a medida que la región experimentaba su primer «ciclo de globalización». ${ }^{6}$ Por último, aunque no disponemos de estudios exhaustivos sobre la distribución de la renta en las décadas centrales del siglo, existen buenas razones para creer que la desigualdad de rentas también cambió considerablemente durante este periodo (Thorp, 1998).

Este artículo pretende por tanto ofrecer las primeras estimaciones coherentes (entre países y a lo largo del tiempo) de desigualdad de renta para la región durante todo el siglo XX, en línea con la hipótesis de Kuznets. El enfoque es una continuación de nuestra labor previa de análisis sobre las tendencias a largo plazo en los niveles de producción, la productividad sectorial y los niveles de vida en la región realizada como parte del proyecto OxLAD 7 (Astorga, Berges y FitzGerald; 2003, 2005a, 2005b). El enfoque adoptado en este trabajo se basa en el pionero de Kuznets $(1955,1959)$, que por una parte relaciona el cambio de la distribución de la renta durante el desarrollo económico con el desplazamiento de la mano de obra entre sectores de producción y niveles de cualificación y, por otra, lo sitúa en un marco de contabilidad nacional de renta agregada. Aparte de la ventaja de no precisar de encuestas domésticas, este enfoque permite interpretar directamente los cambios de la desigualdad en relación con otras variables económicas agregadas.

En sí mismo, el modelo de Kuznets ${ }^{8}$ implica que, durante la industrialización, la distribución de la renta empeora en un principio, al surgir un sector industrial que requiere una elevada cualificación dentro de una economía agrícola en la que la cualificación es baja, pero mejora más tarde cuando el grueso de la mano de obra se desplaza de ésta a aquél. Existen sin embargo al menos dos teorías enfrentadas sobre el cambio de la desigualdad de la renta. Por una parte se encuentra el modelo $\mathrm{H}-\mathrm{O}$ (Heckscher-Ohlin), que sugiere que para una economía rica en recursos concentrada en pocas manos, como ocurre en América Latina, una mayor apertura iría asociada al empeoramiento de la distribución de la renta. Por otra parte, la teoría keynesiana afirma que los cambios macroeconómicos importantes en la demanda y la inversión afectan al empleo y a los salarios reales, por lo que el boom iría asociado a una mejora en la distribución de la renta y la crisis, a un empeoramiento de dicha distribución.

Curiosamente, las tres teorías implican, una vez modificadas para adaptarse a las características e impactos regionales, un perfil bastante parecido del coeficiente de Gini correspondiente a América Latina a lo largo del siglo XX, aunque por muy dis-

${ }^{6}$ Williamson (1999, 2002); Bértola (2005); Bértola y Williamson (2003).

7 OxLAD es la Base de Datos sobre América Latina de la Universidad de Oxford, una completa fuente de estadísticas económicas y sociales para el periodo comprendido entre 1900 y 2005 . Está disponible a través del sitio Web del servicio de información de las bibliotecas de Oxford (OLIS).

${ }^{8}$ Y, en realidad, el modelo de Lewis (1954). 
tintas razones. El modelo de Kuznets no prediría realmente la habitual U invertida recogida en los libros de texto para América Latina porque el proceso de industrialización se estancó en el último cuarto del siglo y el crecimiento de la mano de obra inundó el sector informal de las urbes, por lo que cabe esperar un perfil en N. El modelo $\mathrm{H}-\mathrm{O}$ implicaría también un perfil en $\mathrm{N}$ al tener en cuenta que la región estaba abriéndose durante las primeras décadas, se cerró tras la Gran Depresión y volvió a abrirse en las dos últimas décadas. Por último, el modelo keynesiano implicaría un empeoramiento en el periodo de entreguerras, ya que la región sufrió depresiones, una mejora con el boom de la sustitución de las importaciones durante y después de la Segunda Guerra Mundial y un último empeoramiento en las dos últimas décadas tras la crisis de la deuda... De nuevo, un perfil en $\mathrm{N}$.

El contenido del artículo se estructura como sigue. El apartado 2 establece la metodología empleada para obtener las estimaciones de la distribución funcional de la renta y la derivación de los coeficientes de Gini, desde el año 2000 hacia atrás. Los resultados se presentan en el apartado 3 y se contrastan con la información disponible sobre la distribución de la renta en la primera y en la última década del siglo. En el apartado 4 se estudia un modelo de MCO que contiene factores determinantes relacionados con las tres teorías anteriormente mencionadas, se muestra la solidez estadística de sus resultados y se indican algunas repercusiones interesantes. En el apartado 5 se exponen las conclusiones.

\section{Metodología}

Tan sólo disponemos de datos sobre renta y gasto familiares correspondientes a las últimas décadas del siglo. Existen datos censales a largo plazo, pero las profesiones y oficios no se diferencian suficientemente o no son homogéneos en distintos lugares y momentos, por lo que no sirven para nuestros fines. Además, tampoco incluyen las rentas. En algunos casos se ha realizado un seguimiento de la composición del mercado laboral y de los salarios en países concretos durante periodos relativamente largos, pero no durante todo el siglo ni de forma que permita las comparaciones entre los distintos países. Williamson $(1999,2002)$ ha sido el primero en calcular los salarios reales en el periodo anterior a la Segunda Guerra Mundial. Al ser los salarios una parte de la renta per cápita, Williamson los considera un indicador a grandes rasgos de la distribución de la renta; sin embargo, no distingue entre los niveles de cualificación de la mano de obra ni toma en cuenta el cambio en la distribución por sectores de la mano de obra en distintos momentos. Bértola $(2005,2006)$ utiliza datos censales de Uruguay y Brasil para representar minuciosamente la composición de la mano de obra por nivel de cualificación y aplica los salarios registrados; pero estos innovadores estudios abarcan tan sólo la primera parte del siglo y no abarca los grupos con mayores rentas. Altimir et al. (2003) usan un análisis mediante microsimulación de las encuestas domésticas realizadas en Buenos Aires durante las últimas tres décadas del siglo para descomponer el impacto de los cambios del 
mercado laboral en la distribución de la renta familiar; sin embargo no existe un estudio de este tipo para otros países o para otras décadas.

En consecuencia, la metodología empleada en el presente artículo es indefectiblemente una aproximación general a la realidad. Tiene la ventaja de utilizar los datos disponibles sobre un largo periodo de tiempo, de forma que se garantizala coherencia en el tiempo y entre distintos países, pero no puede en absoluto sustituir a los estudios exhaustivos a nivel nacional. La población activa se divide en cuatro grupos que, a su vez, son un compendio de las categorías empleadas en el Panorama social publicado cada año por la Comisión Económica para América Latina y el Caribe de la ONU. Estos cuatro grupos se muestran en la tabla1, que resume las estimaciones de la CEPAL para América Latina en su conjunto en el año 2000 y que sirven de base para el modelo. Las principales variables son la fracción $\left(n_{i}\right)$ que representa cada grupo dentro de la población activa y la relación $\left(y_{i}\right)$ entre la renta media de cada grupo y la del conjunto de la población activa.

TABLA 1

EMPLEO Y RENTA POR CATEGORÍA PROFESIONAL, AÑO 2000

\begin{tabular}{|c|l|c|c|c|}
\hline & \multicolumn{1}{|c|}{ Categoría } & $\begin{array}{c}\text { Fracción } \\
\text { de pobl. act. }\left(n_{i}\right)\end{array}$ & $\begin{array}{c}\text { Relación con renta media } \\
\text { de pobl. act. }\left(y_{i}\right)\end{array}$ & $\begin{array}{c}\text { Educación } \\
\text { (años) }\end{array}$ \\
\hline 1 & $\begin{array}{l}\text { Empleados, directivos } \\
\text { y profesionales }\end{array}$ & 0,09 & 3,34 & 11 \\
\hline 2 & Técnicos y administrativos & 0,14 & 1,21 & 8 \\
\hline 3 & $\begin{array}{l}\text { Trabajadores urbanos, } \\
\text { artesanos, etc. }\end{array}$ & 0,41 & 0,85 & 6 \\
\hline 4 & $\begin{array}{l}\text { Trabajadores rurales } \\
\text { y sirvientes domésticos }\end{array}$ & 0,36 & 0,49 & 3 \\
\hline
\end{tabular}

Fuente: ONU/CEPAL: Panorama social 2000.

De la tabla 1 también se concluye que, aparte de la renta, existe una diferencia fundamental entre los grupos: su media de años de formación, que puede tomarse como indicador de la cualificación (y, en términos más generales, del capital humano) asociada a la categoría profesional.

Así pues, tenemos que una distribución funcional de la renta se define como:

$$
\sum_{i} n_{i} y_{i}=1
$$


El modelo se basa, por tanto, en trabajo (sueldos y salarios) y capital (beneficios y rentas). Aunque implícitamente las rentas obtenidas por recursos naturales a título privado pertenecen a los ingresos del grupo 1, no existen índices de la superficie de tierra disponible ni de la relación entre trabajo y arrendamientos, como, por ejemplo, en Bértola y Williamson (2003). Esto se debe a diversas razones. En primer lugar, no existen datos fiables y a largo plazo sobre zonas de tierras de cultivo. En segundo lugar, tampoco disponemos de datos a largo plazo sobre arrendamientos. En tercer lugar, allí donde predomina la población rural, los datos sobre el régimen de propiedad de la tierra también serían fundamentales pero tampoco están disponibles de forma cronológica. Por último, los ingresos por recursos distintos de la tierra (p.ej., la minería y el petróleo) van a parar en gran medida a los inversores extranjeros.

En el proyecto OxLAD se presentan series pertinentes para la clasificación de la población activa por sectores productivos, basadas en censos básicos y en datos construidos estadísticamente. Dichas series permiten estimar la cuota o fracción que representan los cuatro grupos dentro de la población activa $\left(n_{i}\right)$ en 1900-2000, tomando 2000 como año de referencia y aplicando cuatro indicadores (con índices basados en el año 2000) como sigue:

- Grupo 1 (empleados, directivos y profesionales). El indicador es la proporción entre graduados universitarios y el total de personas con educación primaria. ${ }^{9}$ La cantidad de graduados se halla aplicando el método de inventario permanente a los datos sobre las matrículas en educación primaria y terciaria.

- Grupo 2 (técnicos y administrativos). El indicador es la proporción de la población activa de empleados en la industria manufacturera y en la administración pública. Los datos sobre los empleados en la industria manufacturera proceden de los datos censales y los de los empleados en la administración pública se han calculado a partir de los niveles de gasto público.

- Grupo 3 (trabajadores urbanos, artesanos, etc.). Este grupo se calcula como el residuo de los otros tres grupos. No se trata sólo de una conveniencia estadística; más bien pretende reflejar el proceso de la migración interna, en el que el sector urbano «informal» actúa como esponja para el exceso de mano de obra subempleada existente en la economía. ${ }^{10}$

- Grupo 4 (mano de obra rural y sirvientes domésticos). El indicador es la cuota o fracción de la población activa dedicada a labores agrícolas, según los datos censales. En él se incluyen, por supuesto, no sólo los trabajadores agrícolas propiamente dichos, sino también los pequeños agricultores (campesinos) y la mano de obra familiar sin retribución económica. Las mujeres rurales son la principal fuente de sirvientes domésticos. siglo.

${ }^{9}$ Para reflejar el aumento del nivel educativo medio de la mano de obra en general a lo largo del

${ }^{10}$ Bulmer-Thomas (1994). 
Los niveles de renta relativa $\left(y_{i}\right)$ se expresan como la relación entre la renta media de cada grupo y la del conjunto de la población activa. Éstos también se calibran para el año 2000, tomado como año de referencia, mediante los indicadores extraídos del proyecto OxLAD.

- Grupo 1. El porcentaje de renta agregada para el grupo se define como el residuo de las rentas agregadas de los otros tres grupos, dividido por la proporción correspondiente de la población activa, lo que da como resultado el nivel de renta relativa:

$$
y_{1}=\frac{\left\{1-\sum_{2}^{4} n_{i} y_{i}\right\}}{n_{1}}
$$

- Grupo 2. Los niveles de renta de este grupo están vinculados a la tendencia en la productividad no agrícola. Esto a su vez implica que los mercados laborales correspondientes a esta categoría se vacían y que, por tanto, los cambios en las rentas relativas reflejan cambios de productividad. Las tendencias de la productividad a lo largo del siglo se tratan de forma detallada en Hofman (2000) y Astorga, Berges y FitzGerald (2003).

- Grupo 3. Los niveles de renta de este grupo están vinculados a la tendencia de los salarios reales de la mano de obra urbana no cualificada, que en muchos casos es el salario mínimo oficial. Estos datos se han recopilado a partir de series de datos nacionales, se han deflactado mediante el IPC y se han ampliado a la relación PIB-población activa. Esta medida es básicamente la utilizada por Williamson (1999).

- Grupo 4. Los niveles de renta están vinculados al índice de productividad agrícola, lo que implica que existe una diferencia considerable entre los salarios urbanos y rurales a principios de siglo, pero que dicha diferencia va reduciéndose a medida que convergen las productividades de los distintos sectores, en parte como consecuencia del propio proceso migratorio.

En este artículo se utiliza el índice de Gini como medida de la dispersión por diversas razones. En primer lugar, porque se trata de una medida de la desigualdad de rentas ampliamente empleada y comprendida. En segundo lugar, aunque el índice Theil es superior técnicamente, ya que permite separar la dispersión dentro del grupo de la dispersión entre los distintos grupos, dado que no se dispone de esas medidas de dispersión, este índice no supone ventaja alguna. Por último, las funciones logarítmicas normales no aprovecharían al máximo los pocos datos de que disponemos. 
Se ha empleado el «método trapezoidal» de determinación de áreas para estimar el coeficiente de Gini $\left(G_{f}\right)$ para los cuatro grupos a partir de una función spline (que vincula polinomios a partir de puntos fijos) derivada de los datos generados por el modelo ${ }^{11}$.

$$
G_{f}=1-\left[y_{4} n_{4}\left(2-n_{4}\right)+y_{3} n_{3}\left\{2\left(1-n_{4}\right)-n_{3}\right\}+y_{2} n_{2}\left(n_{2}+2 n_{1}\right)+y_{1} n_{1}^{2}\right]
$$

Conviene destacar que este coeficiente de Gini es para los ingresos por categoría profesional. No se trata sólo de la renta procedente del sueldo o del salario, lo que supone una mejora respecto a otras estimaciones, pero tampoco recoge la renta individual y, por tanto, no incluye la dispersión dentro de cada grupo. Sin embargo, algunos trabajos sobre la dispersión dentro de los distintos grupos en América Latina en las últimas décadas muestran que el coeficiente de Gini intracuartílico es mucho más estable que el valor intercuartílico (López y Servan, 2005). Tampoco se trata de rentas familiares porque no disponemos de información sobre los índices de dependencia por grupos de renta, lo que probablemente aumentaría aún más la dispersión. ${ }^{12}$

Así pues, los coeficientes de Gini estimados no coinciden con los coeficientes de Gini para rentas familiares, aunque la dirección de la tendencia y el tipo de fluctuaciones deberían ser indicadores fiables de lo que ocurre con la distribución familiar en su conjunto. Por supuesto, podríamos crear un «pseudocoeficiente de Gini para familias» simplemente suponiendo que la relación entre ambos fuera la misma que en el año de referencia (2000) para el que se ha realizado la representación de la CEPAL, pero no se conseguiría añadir más información de la que ya se dispone y podría resultar engañoso.

\section{Resultados}

La tabla 2 muestra el coeficiente de Gini para los ingresos por cada grupo utilizado en el presente documento para el año de referencia (el 2000), en comparación con el coeficiente de Gini para rentas familiares. En ambos casos se utilizaron los mismos datos del CEPAL. Nuestra medición, relativamente burda, explica en realidad más de la mitad (casi dos tercios de media) de los coeficientes de Gini observados para rentas familiares en todos los casos. En un estudio longitudinal de la

11 Véase Gastwirth y Glauberman (1976).

12 El índice de participación (población activa/población) desciende de forma constante desde el $37 \%$ en 1900 hasta el 33\% en 1970 y luego aumenta hasta el 44\% hacia el año 2000, lo que refleja la transición demográfica, a la que siguió una participación cada vez mayor de la mujer (Astorga, Berges y FitzGerald, 2005). El aumento de los índices de mayor dependencia y la reducción de la pobreza debida a la participación de la mujer (Kuznets, 1976) implicarían que la diferencia entre los coeficientes de Gini para las rentas familiares y funcionales habrían caído a lo largo del siglo. 
distribución de la renta en Buenos Aires y periferia durante el último cuarto de siglo, Altimir et al. (2003) observan en el caso de Argentina una relación entre ingresos y coeficiente de Gini para rentas familiares similar a la de la tabla 2, y concluyen que dicha relación es estable.

TABLA 2

COEFICIENTE DE GINI PARA LOS INGRESOS DEL GRUPO Y PARA RENTAS FAMILIARES, AÑO 2000

\begin{tabular}{|l|c|c|c|c|c|}
\hline & Arg & Bra & Chi & Col & Méx \\
\hline Fam. & 0,566 & 0,640 & 0,559 & 0,575 & 0,542 \\
\hline Ingr. & 0,454 & 0,363 & 0,347 & 0,336 & 0,302 \\
\hline Ingr./fam. & 0,803 & 0,567 & 0,622 & 0,584 & 0,557 \\
\hline
\end{tabular}

En el espacio de que disponemos es imposible reproducir de forma significativa todos los resultados de dichas estimaciones, ya que existen para cada año estimaciones para dos variables $(n, y)$, cuatro grupos $(i)$ y cinco países, lo que da lugar a 4000 observaciones. ${ }^{13}$ No obstante, en la tabla 3 del apéndice se muestran los conjuntos de variables para 1900, 1950 y 2000, que sirven para comprender los cambios estructurales que se produjeron a lo largo del siglo.

Las tendencias comunes más evidentes con el tiempo son el descenso de la mano de obra agrícola $\left(n_{4}\right)$ y el incremento de la urbana no cualificada $\left(n_{3}\right)$. Por otra parte, el empleo cualificado $\left(n_{2}\right)$ parece haber aumentado mucho menos a lo largo del siglo en su conjunto. El primer cambio al menos habría mejorado con el tiempo la distribución de la renta si los coeficientes de ingresos $\left(y_{4}, y_{3}\right)$ se hubieran mantenido estables, como suponen los modelos en la línea de Kuznets. Sin embargo, a lo largo del siglo ha ido disminuyendo la diferencia entre ambos como consecuencia del aumento de la productividad agrícola y del exceso de mano de obra en el sector urbano informal (Astorga, Berges y FitzGerald, 2004). Así pues, la mejora prevista en la estructura de la distribución realmente no se ha producido.

La tabla 4 muestra los coeficientes de Gini calculados para los cinco países al final de cada década. Para eliminar el exceso de ruido se ha tomado una media móvil de cinco años de los coeficientes de Gini calculados. En cuanto a los niveles, Argentina y Chile tienen niveles medios inferiores a los de los otros tres países, aunque en realidad estas diferencias no son tan significativas como pudiera parecer debido a la elevada varianza que se da en todos los casos. Sin embargo, algunos países (Brasil y México) tienen una clara propensión a sufrir mayores fluctuaciones que los demás

\footnotetext{
${ }^{13}$ Las hojas de cálculo pertinentes pueden solicitarse a los autores.
} 
TABLA 3

CUOTAS DE LA POBLACIÓN ACTIVA E ÍNDICES DE INGRESOS POR GRUPO PROFESIONAL: 1900, 1950 Y 2000

\begin{tabular}{|c|c|c|c|c|c|c|c|}
\hline \multicolumn{8}{|l|}{ Datos para 2000} \\
\hline & & & Arg & Bra & Chi & $\mathrm{Col}$ & Méx \\
\hline & $\mathrm{n}$ & 1 & 0,15 & 0,08 & 0,16 & 0,15 & 0,10 \\
\hline & & 2 & 0,20 & 0,14 & 0,17 & 0,12 & 0,14 \\
\hline & & 3 & 0,49 & 0,41 & 0,49 & 0,37 & 0,44 \\
\hline & & 4 & 0,24 & 0,37 & 0,18 & 0,36 & 0,32 \\
\hline & & tot & 1,09 & 1,00 & 1,00 & 1,00 & 1,00 \\
\hline & $\mathrm{v}$ & 1 & 2.91 & 3.77 & 2.78 & 238 & 350 \\
\hline & & 2 & 0,99 & 1,24 & 0,99 & 1,42 & 1,22 \\
\hline & & 3 & 0,58 & 0,93 & 0,61 & 0,71 & 0,76 \\
\hline & & 4 & 0,26 & 0,40 & 0,47 & 0,60 & 0,50 \\
\hline
\end{tabular}

\begin{tabular}{|c|c|c|c|c|c|c|c|}
\hline \multicolumn{8}{|l|}{ Estimaciones para 1950} \\
\hline & & & Arg & Bra & Chi & $\mathrm{Col}$ & Méx \\
\hline & $\mathrm{n}$ & 1 & 0,07 & 0,04 & 0,09 & 0,03 & 0,02 \\
\hline & & 2 & 0,17 & 0,05 & 0,13 & 0,14 & 0,13 \\
\hline & & 3 & 0,36 & 0,18 & 0,42 & 0,41 & 0,35 \\
\hline & & 4 & 0,39 & 0,73 & 0,37 & 0,43 & 0,50 \\
\hline & & tot & 1,00 & 1,00 & 1,00 & 1,00 & 1,00 \\
\hline & $\mathrm{y}$ & 1 & 4,19 & 14,15 & 4,35 & 9,87 & 21,84 \\
\hline & & 2 & 1,02 & 2,17 & 1,17 & 1,52 & 1,45 \\
\hline & & 3 & 1,13 & 1,14 & 0,74 & 0,68 & 0,52 \\
\hline & & 4 & 0,26 & 0,19 & 0,46 & 0,51 & 0,47 \\
\hline
\end{tabular}

\begin{tabular}{|c|c|c|c|c|c|c|c|}
\hline \multicolumn{8}{|l|}{ Estimaciones para 1900} \\
\hline & & & Arg & Bra & Chi & Col & Méx \\
\hline & $\mathrm{n}$ & 1 & 0,06 & 0,03 & 0,08 & 0,03 & 0,02 \\
\hline & & 2 & 0,18 & 0,06 & 0,11 & 0,07 & 0,10 \\
\hline & & 3 & 0,37 & 0,11 & 0,40 & 0,07 & 0,32 \\
\hline & & 4 & 0,40 & 0,79 & 0,41 & 0,83 & 0,56 \\
\hline & & tot & 1,00 & 1,00 & 1,00 & 1,00 & 1,00 \\
\hline & & & & & & & \\
\hline & $\mathrm{y}$ & 1 & 6,00 & 11,76 & 4,67 & 15,10 & 7,19 \\
\hline & & 2 & 1,10 & 2,38 & 1,17 & 2,79 & 1,46 \\
\hline & & 3 & 0,97 & 2,55 & 0,63 & 0,91 & 1,15 \\
\hline & & 4 & 0,21 & 0,22 & 0,59 & 0,33 & 0,60 \\
\hline
\end{tabular}


(especialmente Argentina y Chile). Esto puede deberse a unas estructuras económicas y sociales más estables en éstos últimos.

Curiosamente, existen indicios de una eventual convergencia entre los cinco países. Revisando la tabla 3 se observa que este ciclo de convergencia parece estar causado principalmente por el aumento de la similitud en el empleo por sectores y las estructuras de producción mencionado en Astorga, Berges y FitzGerald (2004), aunque parece que también se ha producido cierta convergencia en ingresos reales relativos (relación entre ingresos reales y PIB per cápita) como resultado de la convergencia de las productividades laborales (o. cit.) y del PIB per cápita (Astorga, FitzGerald y Berges, 2005a) a lo largo del siglo. Es de notar que las desviaciones típicas de las series son de la misma orden de magnitud que la diferencia entre el coeficiente de Gini medio para rentas familiares de América Latina y de Asia: 0,15.

\section{TABLA 4}

COEFICIENTES DE GINI PARA INGRESOS POR GRUPO Y DÉCADA; 1900-2000

\begin{tabular}{|r|c|c|c|c|c|c|c|}
\hline & Arg & Bra & Chi & Col & Méx & med5al & Desv. típ. \\
\hline 1900 & 0,478 & 0,659 & 0,365 & 0,623 & 0,270 & 0,479 & 0,166 \\
\hline 1910 & 0,460 & 0,639 & 0,410 & 0,618 & 0,289 & 0,483 & 0,147 \\
\hline 1920 & 0,463 & 0,684 & 0,334 & 0,617 & 0,353 & 0,490 & 0,156 \\
\hline 1930 & 0,356 & 0,674 & 0,412 & 0,568 & 0,391 & 0,480 & 0,135 \\
\hline 1940 & 0,376 & 0,671 & 0,388 & 0,641 & 0,463 & 0,508 & 0,140 \\
\hline 1950 & 0,381 & 0,686 & 0,389 & 0,404 & 0,483 & 0,469 & 0,128 \\
\hline 1960 & 0,405 & 0,579 & 0,400 & 0,455 & 0,484 & 0,465 & 0,073 \\
\hline 1970 & 0,430 & 0,543 & 0,324 & 0,373 & 0,353 & 0,404 & 0,086 \\
\hline 1980 & 0,472 & 0,337 & 0,270 & 0,443 & 0,153 & 0,335 & 0,130 \\
\hline 1990 & 0,512 & 0,360 & 0,317 & 0,355 & 0,232 & 0,355 & 0,102 \\
\hline 2000 & 0,454 & 0,363 & 0,347 & 0,336 & 0,302 & 0,360 & 0,057 \\
\hline Media & 0,435 & 0,563 & 0,360 & 0,494 & 0,343 & 0,439 & 0,120 \\
\hline Desv. típ. & 0,049 & 0,142 & 0,045 & 0,121 & 0,107 & & \\
\hline
\end{tabular}

El gráfico 1 muestra las grandes fluctuaciones ocurridas a lo largo del siglo y que, claramente, no coinciden en los cinco países. Sin embargo se nota la progresiva convergencia. En dos casos (Brasil y Colombia) se ha producido una mejora considerable durante el siglo; en el caso de Chile, Argentina y México la mejora es mucho más discreta. 


\section{GRÁFICO 1}

\section{COEFICIENTE DE GINI PARA INGRESOS POR GRUPO, 1900-2000}

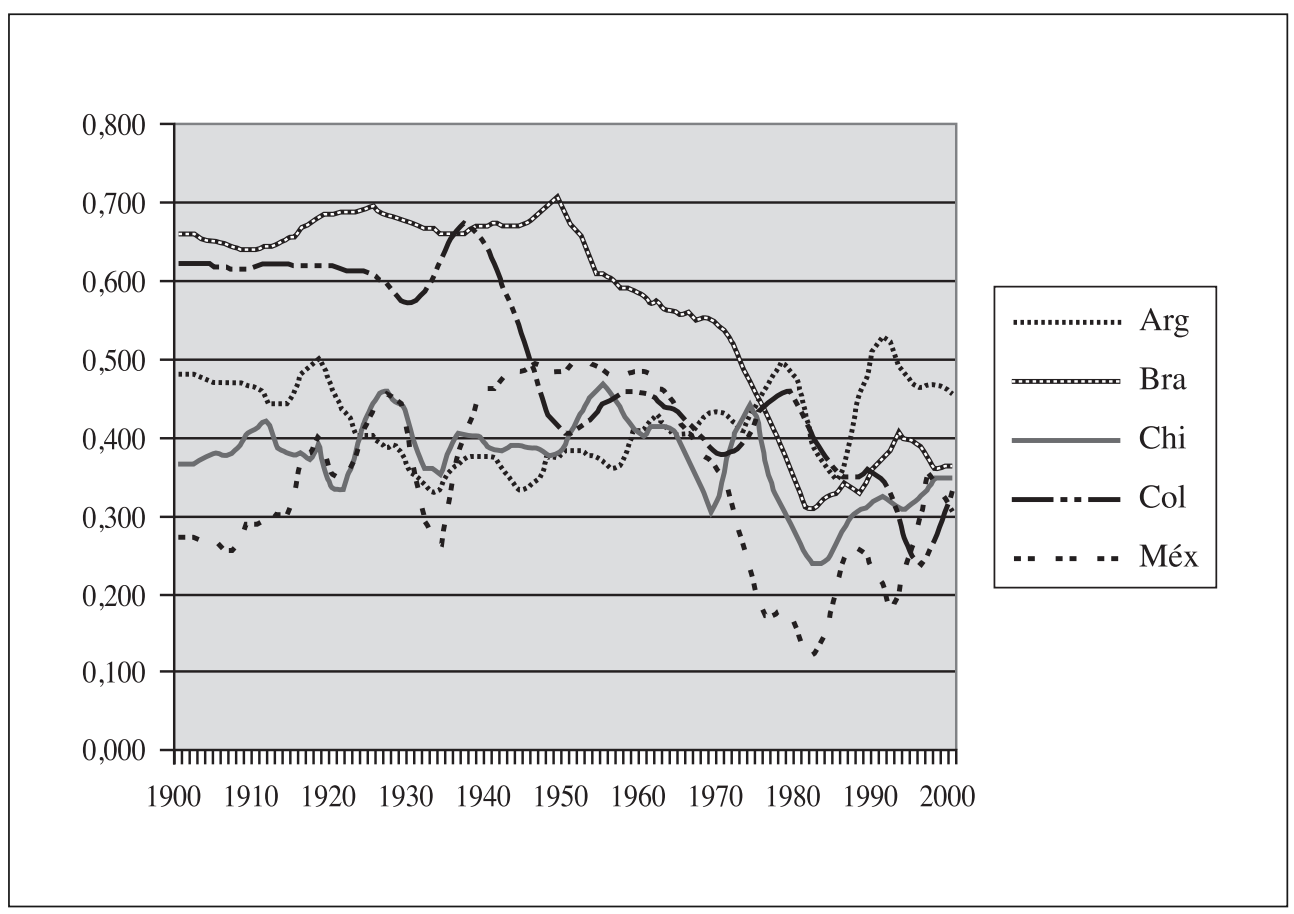

Fuente: elaboración propia.

El gráfico 2 muestra la evolución de los ingresos de la mano de obra urbana no cualificada en relación con el PIB per cápita -a efectos prácticos, el $y_{3}$ del presente estudio- recogidas por Williamson (1999), incluyendo las cuatro primeras décadas del siglo. Williamson (2002) lo llama una «medida de la desigualdad» porque, mientras el numerador tiene en cuenta aspectos relacionados con todos los factores de la producción per cápita de la población, el denominador sólo lo hace con la mano de obra no cualificada, que es el factor más extendido entre la población. Así, Williamson interpreta que los datos reflejan un empeoramiento inicial en la distribución de la renta entre la década de 1880 y la década de 1920, una mejora durante ésta última y, de nuevo, un empeoramiento en la década de 1930. Desafortunadamente, el suyo es el único estudio comparativo y no toma en consideración ningún otro grupo de mano de obra en cuanto a la evolución de sus rentas o al cambio de su peso en el total nacional, como pretende el presente modelo. No obstante, sus conclusiones parecen coherentes con las estimaciones obtenidas (figura1), en parte porque se han utilizado las mismas fuentes primarias para el cálculo de $y_{3}$. 


\section{GRÁFICO 2}

«MEDIDA DE LA DESIGUALDAD» (RELACIÓN ENTRE INGRESOS Y PIB PER CÁPITA, 1913=100)

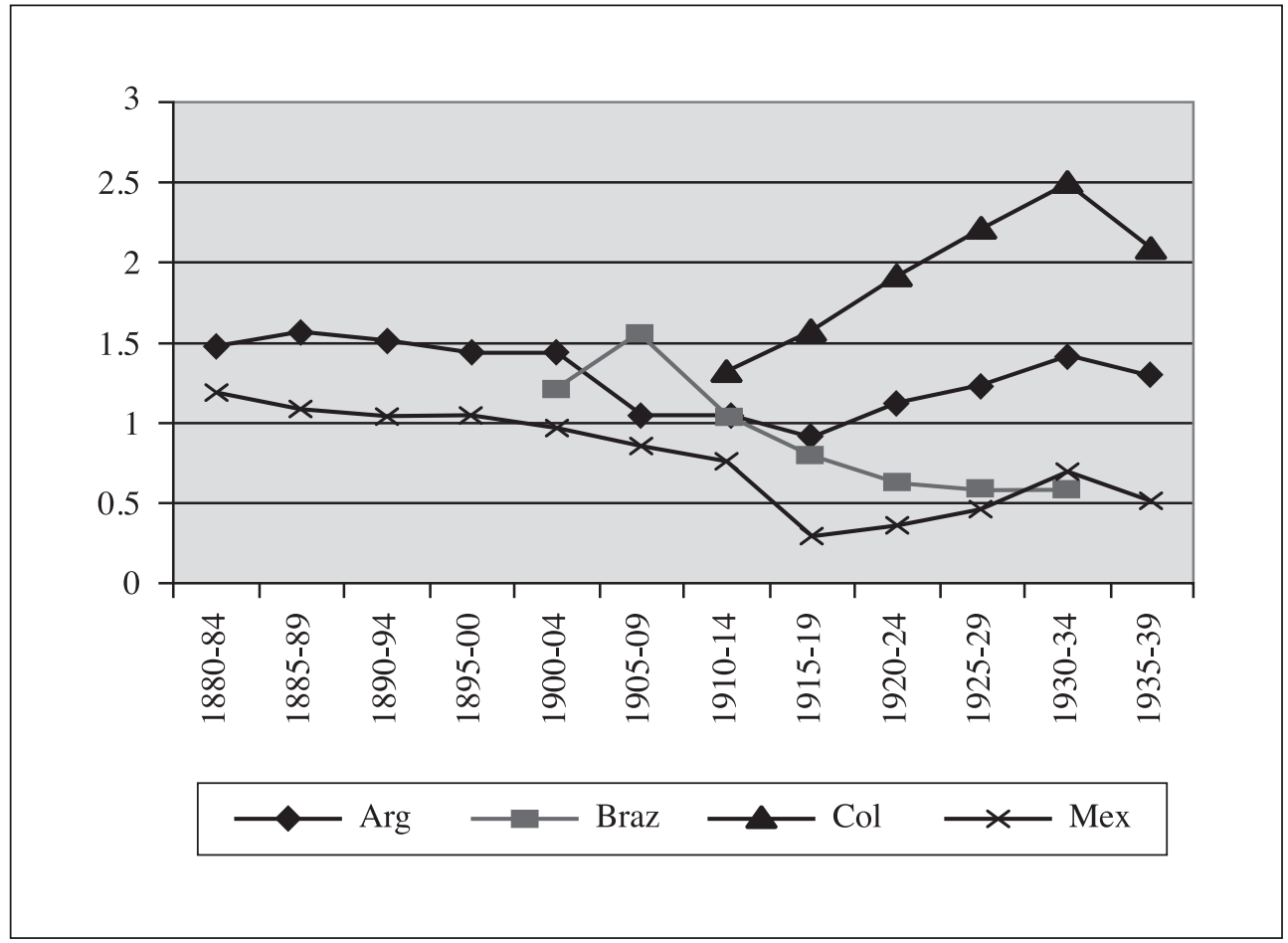

Fuente: Williamson (1999).

El conjunto de datos del BID sobre coeficientes Gini para rentas familiares ${ }^{14}$ es la variable de comparación para las últimas décadas del siglo, aunque no podemos esperar una coincidencia exacta debido, por una parte, a las fluctuaciones en la distribución dentro de los grupos y, por otra, a los impuestos, subsidios y transferencias. En el gráfico 3 se muestran las tendencias de los cuatro países incluidos en la muestra del BID que coinciden con los países objeto de nuestro estudio. Aparte de confirmar la existencia de grandes fluctuaciones, se produce una coincidencia sorprendente en los casos de Brasil, Colombia y México (menor en el caso de Chile) en cuanto a los puntos de inflexión y la dirección de los cambios. ${ }^{15}$

${ }^{14}$ Se trata del único estudio que ha comprobado de forma sistemática la coherencia de las encuestas familiares.

${ }^{15}$ Esto se debe en parte al efecto de los planes de impuestos y traslados aplicados a las rentas familiares, pero el índice oficial de salarios para mano de obra no cualificada también exagera el crecimiento real de los salarios en la década de 1980. 


\section{GRÁFICO 3 \\ ESTIMACIONES DEL COEFICIENTE DE GINI \\ PARA RENTAS FAMILIARES, BID, 1970-1995}

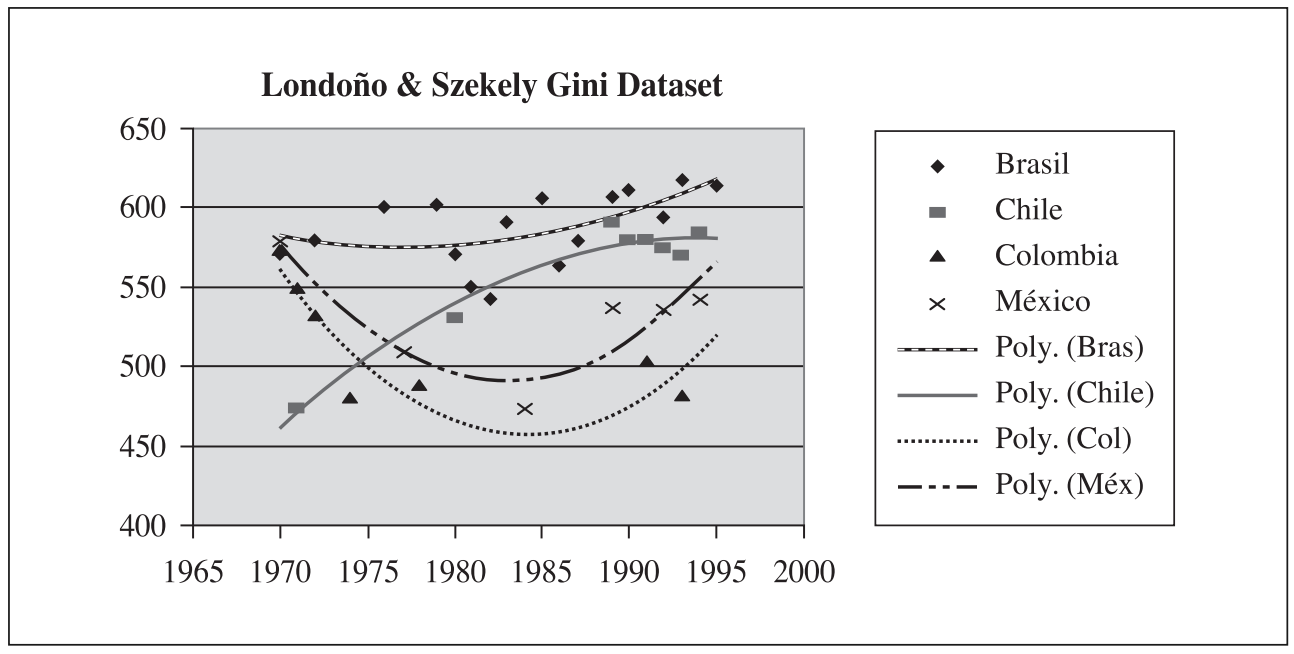

Fuente: Londoño y Szekely (1996). Las líneas de tendencia ajustadas son polinomios cuadráticos.

La estimación hecha por el BID de la evolución del coeficiente de Gini medio de las rentas familiares en América Latina (gráfico 4) en las últimas tres décadas del siglo guarda un parecido en su perfil con al menos la tabla 4 y el gráfico 5 . La varianza a lo largo del ciclo también es la misma $(0,5 \%)$ en ambos estudios para este periodo.

\section{GRÁFICO 4}

COEFICIENTE DE GINI MEDIO PARA AMÉRICA LATINA, BID, 1970-1996

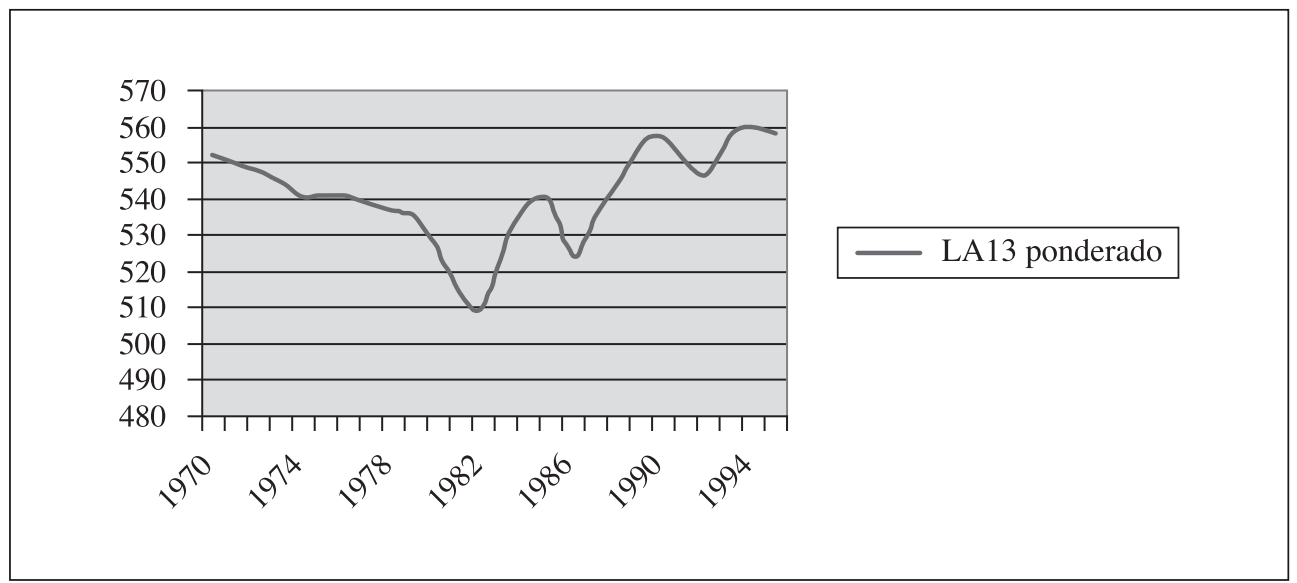

Fuente: Londoño y Szekely (1996). 
Los cálculos realizados en este trabajo no dan como resultado un ciclo claro de distribución de la renta común para los cinco países. No obstante, la media conjunta de este grupo de países ofrece una idea general de todo el continente, como se muestra en el gráfico 5: cierto empeoramiento al comienzo del siglo, seguido de una mejora en la década de 1930 y una marcada mejoría entre las décadas de 1960 y 1980 seguida de un rápido empeoramiento. Dicho de otra manera, un perfil en $\mathrm{N}$ que, además de coincidir con lo que se sabe sobre las primeras y últimas décadas del siglo, concuerda con las predicciones de las tres teorías sobre la distribución de la renta a largo plazo aplicadas a América Latina.

\section{GRÁFICO 5}

COEFICIENTE DE GINI PROMEDIO DEL GRUPO FUNCIONAL DE RENTA LATINOAMERICANO, 1900-2000

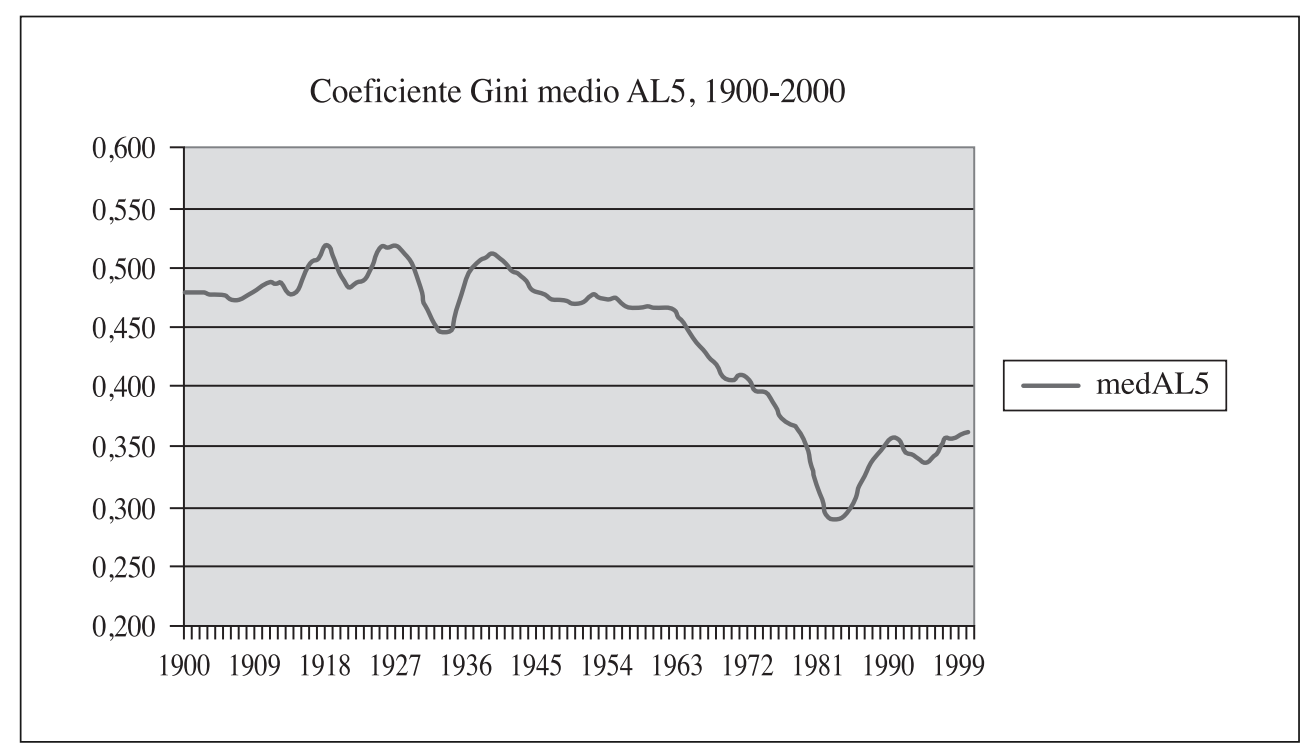

Fuente: elaboración propia.

\section{Análisis}

La estrategia econométrica consistió en emplear un modelo de MCO con EViews, realizando regresiones por separado para cada país y aplicando filtros de HodrickPrescott ${ }^{16}$ a la serie y a las variables independientes del coeficiente de Gini -excep-

${ }^{16}$ El filtro de Hodrick-Prescott (HP) es un filtro lineal bilateral que calcula las series alisadas $s$ de $y$ minimizando la varianza de $y$ en torno a $s$, sujeta a una penalización que limita la segunda diferencia de $s$. 
to cuando ya se habían alisado al construir la serie original-. Estos valores de evolución parecen más adecuados para el estudio de los factores que determinan la desigualdad a largo plazo que para el uso de las variables en su estado original, que están sometidas a un exceso de ruido. ${ }^{17}$ En algunos casos se retardaron las variables independientes uno o más años. Esto se hizo para mejorar la $R^{2}$ corregida y reducir el error típico de la regresión, pero en algunos casos puede tener una justificación económica -como un efecto retardado de la evolución hacia una sociedad urbana o de la apertura de la economía- aunque ya se hayan filtrado las series.

El modelo objeto de estudio tiene nueve factores determinantes, agrupados en tres categorías que reflejan las distintas teorías sobre la determinación de la distribución de la renta, anteriormente comentadas:

- H-O. El índice de tipo de cambio real (ITCR) representa los precios relativos para todo el mundo; no se utilizaron relaciones de comercio exterior, ya que en gran medida guardan correlación con el tipo de cambio real. El índice de apertura -relación de importaciones y exportaciones con el PIB- (APER) refleja la influencia de la integración en los mercados mundiales; mientras que la relación capital-trabajo (CT) representa la dotación de factores y el progreso tecnológico acumulados.

- Kuznets. La urbanización (URB) de la población representa la transformación social: no se puede usar el reparto por sectores de la mano de obra como variable independiente porque ya se ha empleado en el cálculo de los coeficientes de Gini. La proporción estimada de población con educación secundaria (EdS) refleja la cualificación. ${ }^{18}$ La esperanza de vida (VIDA) se usa como representación de otros aspectos de la calidad del capital humano.

- Keynes. La relación capital-producto (CP) refleja la utilización de la capacidad productiva y el rendimiento macroeconómico. La inflación (INF) representa la inestabilidad macroeconómica ${ }^{19}$. La parte del PIB correspondiente al gasto público (GPub) representa la intervención del Estado, pero también el exceso de demanda. El crecimiento del PIB se excluyó de las variables a largo plazo al ser un flujo sujeto a fluctuaciones a corto plazo.

El ajuste es muy bueno en los cinco casos (véase gráfico 6): los coeficientes de regresión corregidos son altos y la gran mayoría de los coeficientes son significativos desde el punto de vista estadístico, como muestra la tabla 5, pero además los

${ }^{17}$ Estos resultados de regresión a corto plazo, en los que se han empleado las variables originales, pueden solicitarse al autor.

${ }^{18}$ Se calcularon a partir de los índices de contratación utilizando el método de inventario permanente, pero dado que los resultados de Brasil y Colombia parecen alcanzar niveles inverosímiles de inestabilidad tras 1960, no se han utilizado hasta que puedan comprobarse las fuentes primarias.

${ }^{19}$ Para solucionar el problema de la hiperinflación (y de sus consecuencias para la convergencia de las regresiones), las series originales se expresaron en forma de ecuación de segundo orden. 


\section{GRÁFICO 6}

\section{TENDENCIAS REALES Y CORREGIDAS DE LOS COEFICIENTES DE GINI}

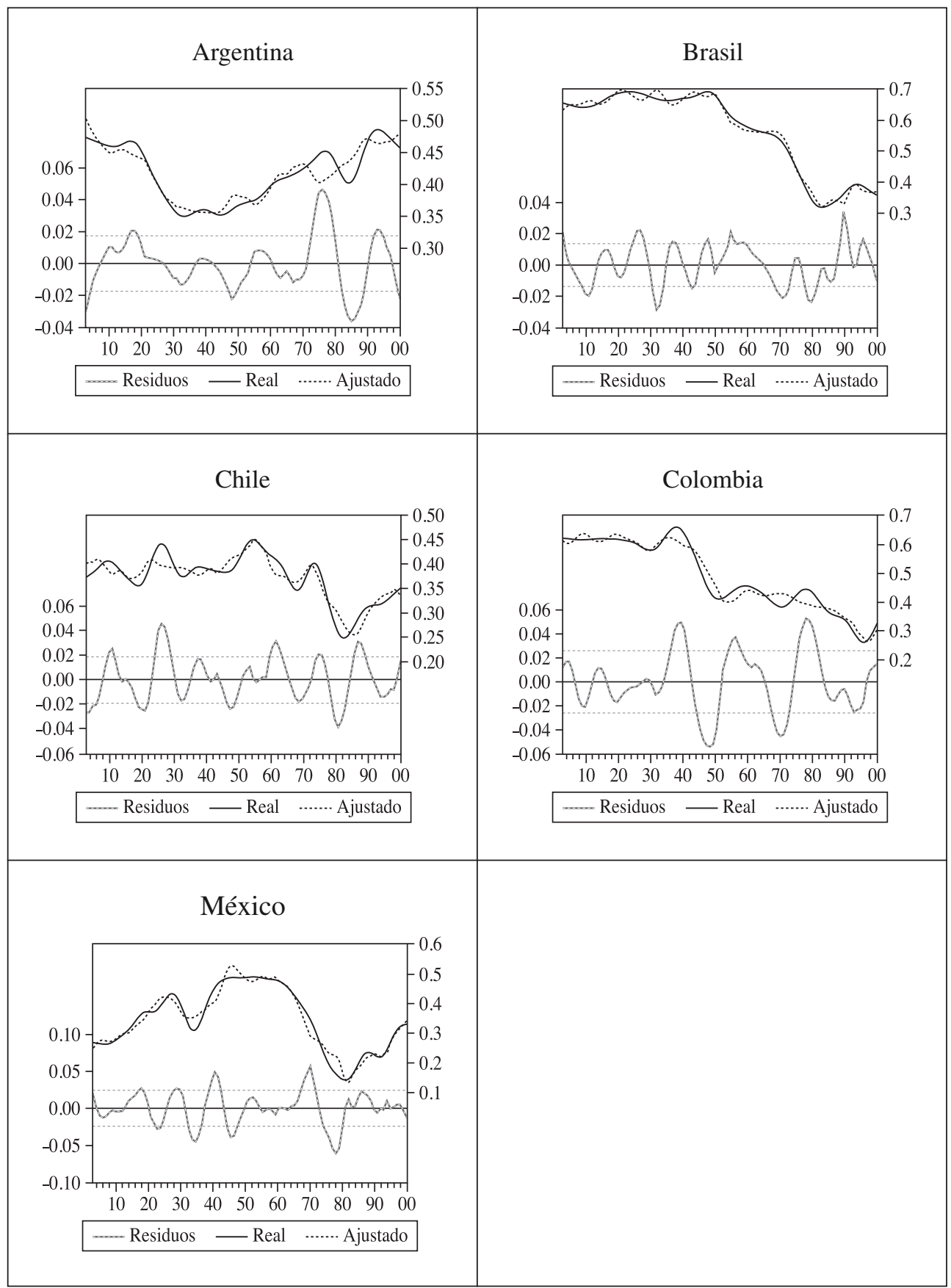

Fuente: elaboración propia. 
estadísticos $\mathrm{F}$ son coherentes (véase el apéndice). Todos los residuos de las regresiones son I(0); es decir, se rechaza la hipótesis del test de Dickey-Fuller aumentado. Este resultado es importante porque significa que las variables fundamentales y los coeficientes de Gini están cointegrados y muestran una relación estable a largo plazo.

Los estadísticos Durbin-Watson (DW) son bajos, pero no queda claro lo que esto significa en el contexto de las regresiones a largo plazo: si los filtros están funcionando correctamente, deberían haber eliminado la mayoría de los componentes cíclicos de la serie. Una explicación podría ser la inercia de la variable dependiente, pero incluso después de añadir un índice de Gini retardado, los consiguientes residuos siguen mostrando una alta correlación. Por último, a primera vista puede intrigar la falta de regularidad en las señales de las variables independientes en los cinco países que se refleja en la tabla 5. Sin embargo, como se verá, esto puede explicarse a la vista de las distintas características estructurales de cada país.

Empezando por el grupo de variables H-O, el tipo de cambio real (ITCR) es significativo en cuatro o cinco casos. Un valor más alto significa una devaluación real, así que en la macroeconomía de una economía abierta típica, debería hundir los salarios reales y estar claramente relacionada con los coeficientes de Gini (FitzGerald, 2007), pero esto sólo ocurre en el caso de Colombia. Una posible explicación es el efecto positivo que tiene el ITCR en los ingresos del sector primario exportador, como sugieren Bourguignon y Morrison (1992). El índice de apertura (APER) es importante en todos los casos: en el caso de América Latina es de esperar que un valor mayor provoque un aumento del coeficiente de Gini debido al efecto sobre las rentas obtenidas por recursos naturales y a la mayor demanda internacional de bienes producidos por mano de obra cualificada que producidos por mano de obra no cualificada (Wood, 1998). Así pues, se esperaba el signo positivo, mientras que el signo negativo de Colombia podría reflejar una larga tradición de políticas de estabilización para mantener el empleo ante posibles impactos externos. La relación capitaltrabajo (CT) es significativa en cuatro de los cinco casos y tiene el efecto esperado de reducir el coeficiente de Gini (presumiblemente a través del efecto de aumentar la productividad de la mano de obra), excepto en el caso de Chile, aunque en este caso el coeficiente es muy pequeño.

Más difícil resulta interpretar el grupo de variables de Kuznets. El grado de urbanización (URB) es importante en todos los casos. Normalmente cabría esperar que esto tuviera un efecto equiparador y que el coeficiente fuera negativo, como ocurre en los casos de Chile, Colombia y México. En el caso de Brasil, que tenía una gran población rural durante la mayor parte del siglo, el signo positivo podría reflejar simplemente el principio del proceso de Kuznets, mientras que para Argentina, que contaba ya con un elevado nivel de urbanización, podría reflejar la pérdida de trabajos rurales de calidad y la migración a las barriadas urbanas. La calidad del capital humano, representado aquí por la esperanza de vida (VIDA), es significativa en cuatro de los cinco casos, pero el signo es desconcertante. ${ }^{20}$ Cabría esperar un coeficiente

\footnotetext{
${ }^{20}$ Como la vida misma...
} 
TABLA 5

COEFICIENTES ESTIMADOS DE LOS DETERMINANTES DEL COEFICIENTE DE GINI

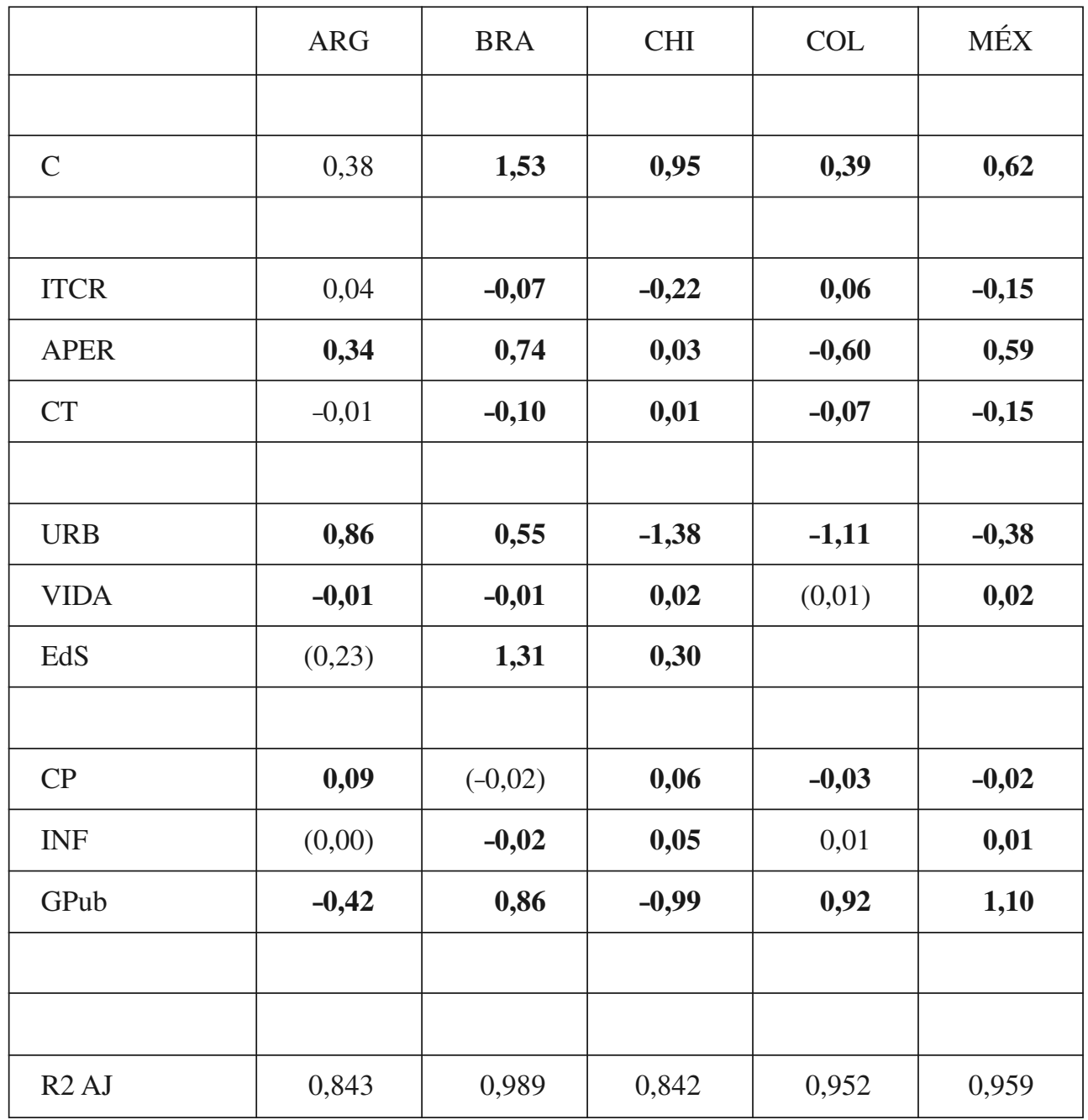

Notas: negrita $=$ significativo al $1 \% ;$ normal $=$ significativo al $5 \% ; \quad($ paréntesis $)=$ no significativo al $10 \%$.

negativo, ya que se trata del indicador aceptado para el bienestar general, pero esto sólo ocurre con Argentina y Brasil. La educación secundaria (EdS) es significativa en dos de los cinco casos y el coeficiente positivo es más lógico de lo que parece si se examina la tabla1, ya que éste se asocia con el crecimiento de la mano de obra cualificada, un grupo minoritario, de modo que en la primera etapa del proceso de Kuznets la distribución de la renta empeorará con su ampliación. 
Por último, el grupo de variables de Keynes también da lugar a resultados interesantes. La relación capital-producto (CP) es significativa en cuatro casos. Los dos coeficientes negativos (Colombia y México) reflejan los beneficios que, como era de esperar, tiene para el empleo la utilización de la capacidad productiva; pero los coeficientes positivos de Argentina y Chile podrían reflejar que dichos beneficios están sesgados de forma asimétrica hacia la mano de obra cualificada. El impacto de la inflación (INF) es significativo en tres de los cinco casos. El signo positivo de Chile y México se corresponden con lo esperado, mientras que el coeficiente negativo de Brasil podría reflejar el efecto más positivo del exceso de demanda nacional en la estimulación del empleo en grandes economías cerradas. Por último, la parte del PIB correspondiente al gasto público (GPub) es significativa en todos los casos. Tan sólo en Argentina y Chile un mayor gasto supone una mejor distribución, pero esto entra dentro de lo esperable, ya que son los países con los sistemas de bienestar más desarrollados. Por contra, en los otros tres casos, los principales beneficiarios podrían ser la minoría de empleados públicos.

\section{Conclusiones}

Del presente artículo pueden extraerse cuatro grandes conclusiones. En primer lugar, que la metodología de Kuznets propuesta para la estimación de la distribución funcional de la renta en ausencia de encuestas domésticas parece útil. Supone un avance respecto al uso de una simple relación entre ingresos de la mano de obra no cualificada y el PIB per cápita para medir los cambios de las desigualdades a largo plazo, ya que abarca cuatro niveles de cualificación cuya representación en la población activa y cuyas rentas relativas varían. Los resultados concuerdan, en términos generales, con la información existente sobre las primeras y últimas décadas del siglo, aunque aún queda mucho por hacer para comprobar las series de los países, en especial en las décadas centrales del siglo.

En segundo lugar, que existen grandes fluctuaciones a lo largo del siglo en el coeficiente de Gini para las rentas, así como una lenta evolución a la baja, por lo que parece que no está justificado el determinismo histórico de algunas publicaciones institucionalistas recientes. Es más, estas fluctuaciones y tendencias pueden explicarse en gran medida mediante variables económicas habituales y, aunque éstas a su vez (como la educación secundaria o las relaciones capital-trabajo) están claramente determinadas por factores institucionales, no sería sensato atribuirlas a un legado económico sino más bien a las políticas económicas y sociales actuales.

En tercer lugar, que aunque los impactos de las variables de origen externo sobre la distribución de la renta varían claramente en función del país, pueden observarse algunos puntos en común. El «efecto H-O» de la integración en los mercados mundiales es notable, pero la dirección del cambio varía según la cualificación de la población, el patrón de propiedad de los recursos naturales y el nivel de capitalización (tecnología). El «efecto Kuznets» del cambio de la distribución de la mano de obra 
es real. En el caso de América Latina, la segunda mitad del proceso de transición (cuando debería mejorar la distribución) se ha visto truncada por el desplazamiento de la mano de obra rural al sector urbano informal no cualificado en vez de a la industria manufacturera altamente cualificada. El «efecto Keynes» sobre los impactos macroeconómicos tampoco es sencillo, ya que depende de la reacción de los distintos niveles de cualificación ante la inestabilidad que provocan.

Por último, que por consiguiente la diferencia histórica en la distribución de la renta $-\mathrm{y}$, de hecho, del crecimiento a largo plazo de la producción- entre América Latina y el resto del mundo se debe principalmente al bajo nivel de cualificación de la mano de obra. La tabla 1 es elocuente en este sentido: tres cuartas partes de la mano de obra en el año 2000 tenían una educación de seis o menos años. Esta combinación de exportación de crecimiento basado en la exportación de recursos naturales y de falta de mano de obra cualificada constituye la gran diferencia entre América Latina y Asia Oriental tras la Segunda Guerra Mundial (Wood, 1997). La lección del siglo XX parece ser que la distribución de la renta sólo mejorará si la mayoría de la mano de obra de la región puede estar cualificada y emplearse en una industria competitiva a nivel mundial (en otras palabras, si se dan las condiciones para pasar a la pendiente descendente de la curva de Kuznets).

\section{Referencias}

[1] ACEMOGLU, D., S. JOHNSON and J.A. ROBINSON (2002): "Reversal of Fortune: Geography and Institutions in the Making of the Modern World Income Distribution", Quarterly Journal of Economics 117, 4: 1231-94.

[2] ALTIMIR, O, L. BECCARIA and M. GONZALEZ-ROZADA (2003): Income distribution in Argentina 1974-2000, Cepal Review 78, pp. 53-82.

[3] ALTIMIR, O. (1987): "Income Distribution Statistics in Latin America and their Reliability", Review of Income and Wealth 33, 2.

[4] ASTORGA, P., A. BERGES and V. FITZGERALD (2003): 'Productivity Growth in Latin America during the Twentieth Century' Discussion Papers in Economic and Social History No 52, Oxford University.

[5] ASTORGA, P., A. BERGES and V. FITZGERALD (2005a): 'The Standard of Living in Latin America during the Twentieth Century' Economic History Review (November 2005).

[6] ASTORGA, P., A. BERGES and V. FITZGERALD (2005b): 'Endogenous Growth and Exogenous Shocks in Latin America during the $\mathrm{XX}^{\text {th }}$ Century' Discussion Papers in Economic and Social History No 57, Oxford University.

[7] BÉRTOLA, L. and J.G. WILLIAMSON (2003): Globalization in Latin America before 1940 NBER Working Paper 9687 Cambridge MA: National Bureau of Economic Research [http://www.nber.org/papers/w9687].

[8] BÉRTOLA, L., C. CASTELNOVO, E. REIS and H. WILLEBALD: An exploration into the distribution of income in Brazil, 1839-1939. Paper presented to XIV International Economic History Congress, Helsinki, 2006. 
[9] BÉRTOLA-FLORES, L. (2005): The Kuznets curve 50 years later: Economic growth and income distribution in Uruguay and other settler economies since 1870 Investigaciones de historia económica, número 3, pp. 135-176.

[10] BOURGUIGNON, F. and C. MORRISON (1992): Adjustment with Equity in Developing Countries, Paris: OECD.

[11] BOURGUIGNON, F. and C. MORRISSON (2002): "Inequality among World Citizens", American Economic Review 92, 4: 727-44.

[12] BULMER-THOMAS, V. (1994): The Economic History of Latin America since Independence, Cambridge: Cambridge University Press.

[13] DE FERRANTI, D., G.E. PERRY, F. FERREIRA and M. WALTON (2004): Inequality in Latin America: Breaking with History? Washington DC: World Bank.

[14] DEININGER, K. and L. SQUIRE (1996): "A New Data Set Measuring Income Inequality”, World Bank Economic Review 10, 3, pp. 565-591.

[15] ENGERMAN, S.L. and K.L. SOKOLOFF (1997): "Factor Endowments, Institutions, and Differential Paths of Growth Among New World Economies", in S. Haber (ed.), How Latin America Fell Behind. Essays on the Economic Histories of Brazil and Mexico, 1800 1914, Stanford: Stanford University Press, pp. 260-304.

[16] ENGERMAN, S.L., S.H. HABER and K.L. SOKOLOFF (2000): 'Inequality, Institutions, and Differential Paths of Growth among New World Economies', in C. Menard (ed.), Institutions, Contracts, and Organizations, Cheltenham: Elgar, pp. 108-34.

[17] FITZGERALD, V. (2007): 'Stabilisation policy and structural adjustment' Chapter 53 in A.K. Dutt and J. Ros (eds.), International Handbook of Development Economics (Vol. II) Northampton, MA: Edward Elgar, in press.

[18] HOFMAN, A.A. (2000): The Economic Development of Latin America in the Twentieth Century, Cheltenham: Elgar.

[19] GASTWIRTH, J.L. and M. GLAUBERMAN (1976): 'The interpolation of the Lorenz Curve and the Gini Index from Grouped Data' Econometrica, 44, pp. 479-483.

[20] KUZNETS, S. (1955): 'Economic growth and income inequality' American Economic Review 45(1), pp. 1-28.

[21] KUZNETS, S. (1959): Quantitative aspects of the growth of nations: IV. Distribution of national income by factor shares' Economic Development and Cultural Change 7 (3,2), pp. 1-100.

[22] KUZNETS, S. (1976): 'Demographic aspects of the size distribution of income: an exploratory essay’ Economic Development and Cultural Change 25 (1), pp. 1-94.

[23] LEWIS, W.A. (1954): "Economic Development with Unlimited Supplies of Labour", Manchester School of Economic and Social Studies 22: 139-91.

[24] LI, H., L. SQUIRE and H. ZOU (1998): Explaining international and intertemporal variations in income inequality, Economic Journal vol. 108(446), pp. 26-43.

[25] LONDOÑO, J.L. and M. SZEKELY (1997): Persistent poverty and excess inequality: Latin America, 1970-95 Working Paper 357, Washington DC: Inter-American Development Bank.

[26] MILANOVIC, B. (2005): Worlds Apart: Measuring International and Global Inequality Princeton NJ: Princeton University Press.

[27] MORLEY, S. (2000): La Distribución del Ingreso en América Latina y el Caribe, Santiago de Chile: Fondo de Cultura Económica and Comisión Económica para América Latina y el Caribe (CEPAL). 
[28] PSACHAROPOULOS, G., S. MORLEY, A. FISZBEIN, H. LEE and B. WOOD (1997): Poverty and Income Distribution in Latin America: the story of the 1980s World Bank Technical Papers 351 Washington DC: World Bank.

[29] SOKOLOFF, K. and S.L. ENGERMAN (2000): "Institutions, Factor Endowments, and Paths of Development in the New World", Journal of Economic Perspectives, 14, 3: 217-32.

[30] THORP, R. (1998): Progress, Poverty and Exclusion. An Economic History of Latin America in the 20th Century, Washington: Inter-American Development Bank.

[31] WILLIAMSON, J.G. (1999): "Real Wage Inequality and Globalization in Latin America before 1940", Revista de Historia Económica XVII (special issue), pp. 101-42.

[32] WILLIAMSON, J.G. (2002): "Land, Labor, and Globalization in the Third World, 18701940", Journal of Economic History 62, 1: 55-85.

[33] WOOD, A. (1997): Openness and Wage Inequality in Developing Countries: The Latin American Challenge to East Asian Conventional Wisdom World Bank Economic Review 11 (1), pp. 33-57.

[34] WOOD, A. (1998): Globalisation and the Rise in Labour Market Inequalities The Economic Journal, Vol. 108, No. 450, pp. 1463-1482. 


\section{ARGENTINA}

Variable dependiente: AGINI_HP

Método: mínimos cuadrados

Fecha: 11/11/07 Hora: 15:31

Muestra (corregida): 19032000

Observaciones incluidas: 98 tras corregir puntos finales

\begin{tabular}{|l|c|c|c|c|}
\hline Variable & Coeficiente & Error típico & t de Student & Prob. \\
\hline C & 0,381 & 0,171 & 2,23 & 0,028 \\
\hline AITCRC_HP(-2) & 0,042 & 0,020 & 2,05 & 0,044 \\
\hline AAPER_HP & 0,336 & 0,129 & 2,61 & 0,011 \\
\hline AURB(-1) & 0,860 & 0,068 & 12,69 & 0,000 \\
\hline AVIDA & $-0,014$ & 0,002 & $-6,75$ & 0,000 \\
\hline ACP_HP & 0,088 & 0,020 & 4,43 & 0,000 \\
\hline ACT(-3) & $-0,014$ & 0,007 & $-2,11$ & 0,038 \\
\hline ATINF_HP(-1) & $-0,002$ & 0,002 & $-1,27$ & 0,209 \\
\hline AGPub_HP & $-0,428$ & 0,120 & $-3,58$ & 0,001 \\
\hline AEdS_HP & 0,234 & 0,194 & 1,21 & 0,230 \\
\hline & & & & 0,417 \\
\hline R cuadrado & 0,858 & \multicolumn{2}{|l|}{ Var. dep. media: } & 0,044 \\
\hline R cuadrado corregida & $\mathbf{0 , 8 4 3}$ & Desv. típ. de var. dep. & $-4,903$ \\
\hline Err. típ. de regresión & $\mathbf{0 , 0 1 7}$ & \multicolumn{2}{l|}{ Criterio de información de Akaike } & $-5,167$ \\
\hline Suma cuadrado de residuos & 0,027 & Criterio de Schwarz & 5,036 \\
\hline Logaritmo de verosimilitud & 263,182 & Estadístico F & \\
\hline Estadístico Durbin-Watson & 0,125 & Prob (estadístico F) & \\
\hline
\end{tabular}

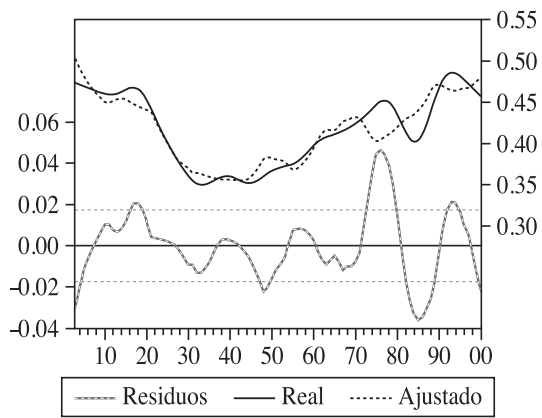




\section{BRASIL}

Variable dependiente: BGINI_HP

Método: mínimos cuadrados

Fecha: 11/11/07 Hora: 15:03

Muestra (corregida): 19032000

Observaciones incluidas: 98 tras corregir puntos finales

\begin{tabular}{|c|c|c|c|c|}
\hline Variable & Coeficiente & Error típico & $\mathrm{t}$ de Student & Prob. \\
\hline $\mathrm{C}$ & 1,533 & 0,075 & 20,37 & 0,000 \\
\hline BITCRC_HP & $-0,065$ & 0,009 & $-6,92$ & 0,000 \\
\hline BAPER_HP & $-0,739$ & 0,083 & $-8,86$ & 0,000 \\
\hline BURB & 0,547 & 0,078 & 7,04 & 0,000 \\
\hline BVIDA & $-0,013$ & 0,001 & $-10,66$ & 0,000 \\
\hline BCP_HP(-3) & $-0,018$ & 0,011 & $-1,60$ & 0,112 \\
\hline BCT $(-1)$ & $-0,101$ & 0,007 & $-14,16$ & 0,000 \\
\hline BTINF_HP & $-0,021$ & 0,002 & $-13,92$ & 0,000 \\
\hline BGPub_HP & 0,855 & 0,108 & 7,90 & 0,000 \\
\hline $\mathrm{R}$ cuadrado & 0,990 & \multicolumn{2}{|l|}{ Var. dep. media: } & 0,564 \\
\hline $\mathbf{R}$ cuadrado corregida & 0,989 & \multicolumn{2}{|c|}{ Desv. típ. de var. dep. } & 0,131 \\
\hline Err. típ. de regresión & 0,014 & \multicolumn{2}{|c|}{ Criterio de información de Akaike } & $-5,667$ \\
\hline Suma cuadrado de residuos & 0,017 & \multicolumn{2}{|c|}{ Criterio de Schwarz } & $-5,430$ \\
\hline Logaritmo de verosimilitud & 286,703 & \multicolumn{2}{|l|}{ Estadístico F } & 1105,271 \\
\hline Estadístico Durbin-Watson & 0,305 & \multicolumn{2}{|c|}{ Prob (estadístico F) } & 0,000 \\
\hline
\end{tabular}

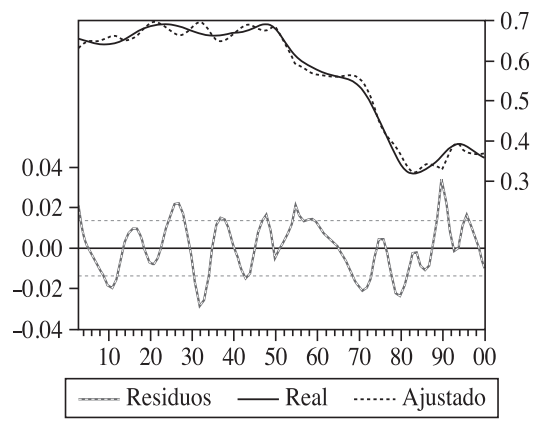




\section{CHILE}

Variable dependiente: CHGINI_HP

Método: mínimos cuadrados

Fecha: 11/11/07 Hora: 15:33

Muestra (corregida): 19042000

Observaciones incluidas: 97 tras corregir puntos finales

\begin{tabular}{|l|c|c|c|c|}
\hline Variable & Coeficiente & Error típico & t de Student & Prob. \\
\hline C & 0,945 & 0,050 & 18,92 & 0,000 \\
\hline CHITCRC_HP & $-0,221$ & 0,024 & $-9,17$ & 0,000 \\
\hline CHAPER_HP & 0,029 & 0,065 & 0,45 & 0,657 \\
\hline CHURB(-2) & $-1,377$ & 0,206 & $-6,68$ & 0,000 \\
\hline CHVIDA(-4) & 0,015 & 0,003 & 6,01 & 0,000 \\
\hline CHCP_HP(-3) & 0,055 & 0,015 & 3,65 & 0,000 \\
\hline CHCT(-1) & 0,014 & 0,004 & 3,97 & 0,000 \\
\hline CHTINF_HP & 0,050 & 0,005 & 9,41 & 0,000 \\
\hline CHGPub_HP & $-0,985$ & 0,088 & $-11,17$ & 0,000 \\
\hline CHEdS_HP & 1,308 & 0,256 & 5,11 & 0,000 \\
\hline & & & & 0,371 \\
\hline R cuadrado & 0,857 & \multicolumn{2}{|l|}{ Var. dep. media: } & 0,048 \\
\hline R cuadrado corregida & $\mathbf{0 , 8 4 2}$ & Desv. típ. de var. dep. & $-4,718$ \\
\hline Err. típ. de regresión & $\mathbf{0 , 0 1 9}$ & \multicolumn{2}{l|}{ Criterio de información de Akaike } & $-4,983$ \\
\hline Suma cuadrado de residuos & 0,032 & Criterio de Schwarz & 57,840 \\
\hline Logaritmo de verosimilitud & 251,675 & Estadístico F & \\
\hline Estadístico Durbin-Watson & 0,235 & Prob (estadístico F) & \\
\hline
\end{tabular}

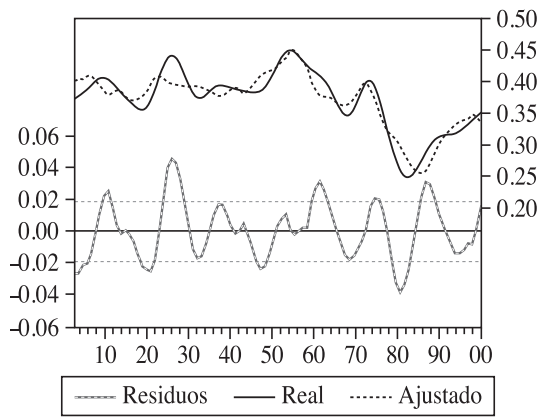




\section{COLOMBIA}

Variable dependiente: CGINI_HP

Método: mínimos cuadrados

Fecha: 11/11/07 Hora: 15:11

Muestra (corregida): 19022000

Observaciones incluidas: 99 tras corregir puntos finales

\begin{tabular}{|l|c|c|c|c|}
\hline Variable & Coeficiente & Error típico & t de Student & Prob. \\
\hline C & 0,387 & 0,229 & 1,69 & 0,094 \\
\hline CITCRC_HP & 0,062 & 0,022 & 2,83 & 0,006 \\
\hline CAPER_HP & $-0,601$ & 0,188 & $-3,19$ & 0,002 \\
\hline CURB(-1) & $-1,117$ & 0,273 & $-4,10$ & 0,000 \\
\hline CVIDA & 0,010 & 0,005 & 1,81 & 0,074 \\
\hline CCP_HP(-2) & $-0,033$ & 0,010 & $-3,18$ & 0,002 \\
\hline CCT & $-0,067$ & 0,026 & $-2,58$ & 0,011 \\
\hline CTINF_HP(-1) & 0,008 & 0,004 & 1,92 & 0,058 \\
\hline CGPub_HP & 0,915 & 0,306 & 2,99 & 0,004 \\
\hline & & & & 0 \\
\hline R cuadrado & 0,956 & \multicolumn{2}{|l}{ Var. dep. media: } & 0,489 \\
\hline R cuadrado corregida & $\mathbf{0 , 9 5 2}$ & Desv. típ. de var. dep. & 0,120 \\
\hline Err. típ. de regresión & $\mathbf{0 , 0 2 6}$ & Criterio de información de Akaike & $-4,351$ \\
\hline Suma cuadrado de residuos & 0,062 & Criterio de Schwarz & $-4,115$ \\
\hline Logaritmo de verosimilitud & 224,374 & Estadístico F & 241,730 \\
\hline Estadístico Durbin-Watson & 0,136 & Prob (estadístico F) & 0,000 \\
\hline & & & \\
\hline
\end{tabular}

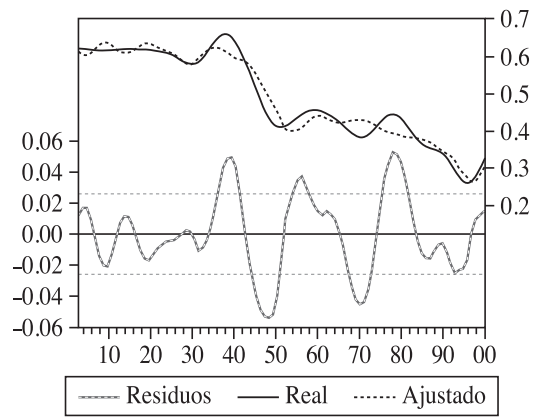




\section{MÉXICO}

Variable dependiente: MGINI_HP

Método: mínimos cuadrados

Fecha: 11/11/07 Hora: 15:24

Muestra (corregida): 19022000

Observaciones incluidas: 99 tras corregir puntos finales

\begin{tabular}{|l|c|c|c|c|}
\hline Variable & Coeficiente & Error típico & Estadístico T & Prob. \\
\hline C & 0,621 & 0,096 & 6,46 & 0,000 \\
\hline MITCRC_HP & $-0,152$ & 0,020 & $-7,61$ & 0,000 \\
\hline MAPER_HP & 0,585 & 0,105 & 5,56 & 0,000 \\
\hline MURB & $-0,377$ & 0,168 & $-2,24$ & 0,027 \\
\hline MVIDA & 0,019 & 0,003 & 6,76 & 0,000 \\
\hline MCP_HP(-2) & $-0,024$ & 0,010 & $-2,50$ & 0,014 \\
\hline MCT & $-0,151$ & 0,009 & $-17,43$ & 0,000 \\
\hline CTINF_HP(-1) & 0,011 & 0,003 & 3,16 & 0,002 \\
\hline MGPub_HP & 1,099 & 0,153 & 7,16 & 0,000 \\
\hline MEdS_HP & 0,297 & 0,132 & 2,25 & 0,027 \\
\hline & & & & 0,346 \\
\hline R cuadrado & 0,953 & \multicolumn{2}{|l|}{ Var. dep. media: } & 0,106 \\
\hline R cuadrado corregida & $\mathbf{0 , 9 4 9}$ & Desv. típ. de var. dep. & $-4,265$ \\
\hline Err. típ. de regresión & $\mathbf{0 , 0 2 4}$ & Criterio de información de Akaike & $-4,527$ \\
\hline Suma cuadrado de residuos & 0,051 & Criterio de Schwarz & 201,702 \\
\hline Logaritmo de verosimilitud & 234,090 & Estadístico F & \\
\hline Estadístico Durbin-Watson & 0,254 & Prob (estadístico F) & \\
\hline
\end{tabular}

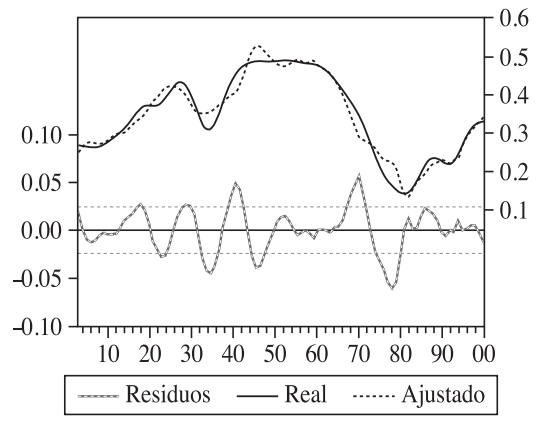

